\title{
Modeling Gas Nucleation and Condensation Using the Direct Simulation Monte Carlo Method
}

\author{
Quanhua Sun ${ }^{*}$ and Iain D. Boyd ${ }^{\dagger}$ \\ University of Michigan, Ann Arbor, MI 48109
}

\begin{abstract}
A general kinetic nucleation and condensation model is proposed and implemented in the direct simulation Monte Carlo method. Both gas and clusters are treated as particles in this model, and clusters possess internal energy and potential energy. Nucleation and condensation processes are treated as collision events, and the probability for possible nucleation or condensation is determined from the nucleation rate and sticking coefficient. Literature review shows that there are many uncertainties in gas nucleation and condensation theory. The proposed model is then very useful for parametric studies. Our parametric studies show that both nucleation rate and sticking coefficient affect properties of the gas and clusters. Simulation of supersonic flow in an expansion nozzle shows that the proposed model is able to simulate complicated flows involving clusters.
\end{abstract}

\section{Nomenclature}

$\begin{array}{ll}c & =\text { constant } \\ C_{p} & =\text { specific heat } \\ E & =\text { energy } \\ G & =\text { free energy } \\ h_{f g} & =\text { enthalpy change during condensation } \\ J & =\text { nucleation rate } \\ j & =\text { cluster size } \\ k & =\text { Boltzmann constant } \\ m & =\text { molecular mass } \\ n & =\text { number density } \\ P & =\text { pressure of vapor, or probability } \\ P_{s} & =\text { saturated vapor pressure } \\ Q & =\text { heat } \\ q_{s} & =\text { sticking coefficient } \\ r & =\text { cluster radius } \\ S & =\text { degree of saturation } \\ T & =\text { temperature } \\ V & =\text { velocity } \\ x, y & =\text { variables } \\ v & =\text { temperature index of viscosity } \\ \rho_{l} & =\text { mass density of liquid } \\ \rho_{v} & =\text { mass density of vapor } \\ \sigma & =\text { surface tension } \\ \sigma_{T} & =\text { collision cross section } \\ \xi & =\text { number of degrees of freedom } \\ & \end{array}$

\footnotetext{
* Research Fellow, Department of Aerospace Engineering, 1320 Beal Avenue, Senior Member AIAA.

${ }^{\dagger}$ Professor, Department of Aerospace Engineering, 1320 Beal Avenue, Associate Fellow AIAA.
} 


\section{Introduction}

$\mathrm{I}_{\mathrm{t} i \operatorname{sen}}^{\mathrm{n}}$ recent years there has been much interest in the behavior of clusters. The formation and growth of clusters through gas nucleation and condensation is believed essential to many phenomena. For instance, vapor-liquid nucleation processes play an important role in the formation of atmospheric aerosols. ${ }^{1}$ The formation of soot begins with formation of clusters. ${ }^{2}$ Gas condensation can also occur within thruster plumes, and clusters may cause contamination of the rocket surfaces. ${ }^{3}$ The formation of clusters will affect the supersonic flow in expansion nozzles due to the heat released during the phase change process. ${ }^{4}$ Cluster formation and growth is also involved in many material processes, including chemical vapor deposition, ${ }^{5}$ dry etching, ${ }^{6}$ and nanoscale material manufacturing. ${ }^{7}$ Understanding the mechanism of the gas nucleation (cluster formation) and condensation (cluster growth) will therefore help improve fabricating techniques and the quality of the resultant products.

Studies on gas nucleation and condensation are generally based on two approaches: continuum approach and kinetic approach. The continuum approach is usually called classical nucleation theory ${ }^{8,9}$ that is derived from thermodynamics. It is assumed that homogeneous nucleation starts when the free energy loss from the transition of gas molecules into the liquid phase can compensate for the energy increase resulting from the surface tension of a cluster. Different expressions have been derived for the nucleation rate, and some of them can differ by a factor of $10^{17}$ for the magnitude of the nucleation rate. ${ }^{9}$ Furthermore, the surface tension of clusters is still unclear yet. If the value of the bulk material is used for the surface tension of clusters, an advanced expression (Lothe and Pound ${ }^{10}$ ) for the nucleation rate predicts a much worse nucleation rate than a simple expression (Becker and Doring ${ }^{8}$ ) as compared with measurement data. Therefore there are still many uncertainties in the classical nucleation theory. On the other hand, many efforts have been made recently to develop kinetic approaches. One is called Smoluchowski's approach where nucleation is viewed as a process of chemical aggregation. ${ }^{11}$ The key issue is to determine the rate constants that are still unresolved yet. A fundamental approach for studying nucleation and condensation is the molecular dynamics method that simulates molecular movement and interactions directly. ${ }^{12}$ This approach is supposed to expose the detailed nucleation and condensation processes, and has been applied to study gas nucleation and condensation. ${ }^{8,13-16}$ However, studies using molecular dynamics are limited to systems with few clusters or/and clusters having small size because of the large numerical cost for simulations. It is impractical to simulate mesoscale systems such as supersonic flow in an expansion nozzle based on the current computational capability.

A more efficient kinetic approach, the direct simulation Monte Carlo (DSMC) method, ${ }^{17}$ has been introduced into the field of gas nucleation and condensation. The DSMC method is less numerically expensive than molecular dynamics but still kinetically accurate, and gas nucleation and condensation models can be easily implemented in the DSMC method. Therefore, the DSMC method is becoming a popular approach for model testing and flow investigation. ${ }^{11,18-22}$ For instance, Hettema and McFeaters ${ }^{11}$ used the DSMC method to implement the Smoluchowski approach; Zhong et al. ${ }^{21}$ employed the classical nucleation theory in the DSMC method studying supersonic plumes. Most of these applications, however, have neglected the cluster effects on gas flows, which then affects the cluster modeling. There are two significant influences of clustering on gas flows: the removal of a portion of the vapor phase and the "heating" of the remainder to absorb the energy extracted from the condensed phase. In the usual case, the ratio $h_{f g} / C_{p} T$ ( $h_{f g}$ being the enthalpy change during condensation, $C_{p}$ being the specific heat, and $T$ is the local temperature) is greater than unity and this means that "heating" has a larger effect on the stream properties than the vapor removal. ${ }^{4}$ These effects were partially demonstrated in the plume studies by Perrell et al. although a continuum-based approach was used. ${ }^{23}$ We also showed the clustering effects on gas flow in our previous paper when studying gas expansion in a supersonic nozzle by including dimers in the simulation. ${ }^{22}$ However, there is no systematic nucleation and condensation model developed for the DSMC method yet.

The main goal of this study is to develop a general nucleation and condensation model to be implemented in the DSMC method. The model itself is not able to predict the value of parameters for simulations, but is supposed to utilize new results from molecular dynamics simulations. The rest of the paper is organized as follows. Brief description of homogeneous nucleation and condensation theory is given in section II. Then microscopic modeling that is suitable for the DMSC method is discussed in section III. Several parametric studies are given in section IV. Finally, the paper ends with some concluding remarks.

\section{Homogeneous Nucleation and Condensation Theory}

A gas is regarded as undersaturated, saturated, or supersaturated if the pressure of the gas $(P)$ is less than, equal to, or larger than the equilibrium saturation value $\left(P_{s}\right)$ corresponding to the local temperature. The undersaturated and saturated states are thermodynamically stable, whereas the supersaturated state is unstable. The supersaturated 
gas will nucleate and condense in the presence of impurities or walls. Otherwise, the gas sustains a very high degree of saturation $\left(S=P / P_{s}\right)$ until homogeneous nucleation occurs.

There are generally two different views on the homogeneous nucleation processes. The classical nucleation theory (CNT) indicates that nucleation begins after the "energy barrier" is overcome; whereas kinetic approaches, such as Smoluchowski's approach, assume that nucleation starts from formation of dimers. In the classical nucleation theory, it is assumed that clusters grow or shrink via the attachment or loss of a single molecule. Making this approximation leads to a set of coupled rate equations for the number density of clusters of different size. Further with the equilibrium state assumption, the nucleation rate $(J)$ can be expressed as follows:

$$
J=\frac{c P\left(4 \pi r_{*}^{2}\right) n}{\sqrt{2 \pi m k T}} \exp \left(-\frac{\Delta G^{*}}{k T}\right)
$$

where $r_{*}$ is the critical cluster radius, and $\Delta G^{*}$ is the free energy of formation of a critical-sized cluster. The other variables are: Boltzmann constant $k$, gas number density $n$, molecular mass $m$, and constant $c$. The critical size is defined such that the free energy of formation reaches the maximum value at this size, and clusters larger than this size are stable whereas smaller clusters are thermodynamically unstable. The CNT relies on a macroscopic approximation for evaluation of the free energy of clusters. This of course makes no sense for small clusters of a few molecules. There are also arguments on the number of degrees of freedom to be included in the evaluation of free energy. ${ }^{9}$ In general, the nucleation rate can be expressed using a nucleation constant $\left(c_{n}\right)$ :

$$
J=c_{n} \sqrt{\frac{2 \sigma}{\pi m^{3}}} \frac{\rho_{v}^{2}}{\rho_{l}} \exp \left(-\frac{4 \pi r_{*}^{2} \sigma}{3 k T}\right)
$$

where $\sigma$ is the surface tension of clusters, and $\rho_{l}, \rho_{v}$ are the mass densities of liquid and vapor, respectively. The critical cluster radius is given as

$$
r_{*}=\frac{2 \sigma}{\rho_{l} R T \ln S}
$$

Determining the value of $\sigma$ is another challenge. There is no general agreement on how the surface tension relates to the radius of curvature. ${ }^{4}$ The surface tension is also temperature-dependent. Although there are several uncertainties in the CNT, measurement data can actually follow the CNT prediction (expression (2)) for most cases. ${ }^{8}$ However, when the vapor temperature is low, the critical size predicted by CNT may not be reasonable. For instance, for condensation of water vapor in air at low temperatures $T<270 K$, the size of the critical cluster determined by CNT is less than the water molecule itself. ${ }^{24}$ Some even suggested not to use the CNT for modeling the onset of clustering in supersonic beams. ${ }^{25}$

Kinetic approaches, however, do not use the concept of "equilibrium state" and there is no critical cluster size. Clusters are formed when two molecules approach each other and form a bound state. It is generally assumed that the formation of a bound state is feasible only when a third particle interacts with one of the two particles and carries away the kinetic energy. Molecular dynamics simulation by Zeifman et al. confirmed that condensation starts from dimer formation in triple collisions of monomers. ${ }^{26}$ The nucleation rate, however, has not been generally determined. Because clusters can dissociate, the cluster distribution can diverge as the volume containing the clusters increases. Treatment of the volume dependence of the free energy has been a source of confusion and controversy in the development of a general molecular theory of nucleation. Other kinetic approaches, however, cannot predict the nucleation rate. For instance, the Smoluchowski approach treats cluster formation as a chemical reaction, but the value of rate constants is unavailable from the approach itself. Another difference is that a third particle is not included for the cluster formation reaction in the Smoluchowski approach. Overall, there is no general expression for the nucleation rate in kinetic approaches.

There are fewer discrepancies about the growth and depletion of clusters. Both continuum and kinetic approaches assume that cluster condensation and evaporation proceeds via one monomer at a time. Other processes such as rearrangement and fragmentation processes are sometimes included. When a monomer has a collision with a cluster, a sticking coefficient $\left(q_{s}\right)$ is used to indicate the possibility for a condensation event. Then the condensation mass flux can be expressed as $q_{s} P / \sqrt{2 \pi R T}$. A collision between a monomer and a cluster can also activate the cluster to evaporate. The evaporation mass flux is derived such that the evaporation mass flux is equal to the condensation mass flux in equilibrium, and is expressed as $q_{s} P_{d} / \sqrt{2 \pi R T_{d}}$, where $T_{d}$ is the cluster temperature and $P_{d}$ is the hypothetical ambient pressure which would be necessary to keep the cluster in equilibrium. 


\section{Microscopic Modeling}

Gas nucleation and condensation are essentially kinetic processes. Detailed description of these processes, however, is numerically too expensive; and it is unnecessary for many practical applications. A statistical description is then more useful for numerical simulations. In this section, we will discuss some properties of clusters, and develop microscopic models that can be easily implemented in the direct simulation Monte Carlo method for gas nucleation and condensation.

\section{A. Some Properties of Clusters}

Theoretically, clusters bridge the gap between microscopic gas and macroscopic materials. The behavior of small clusters (dimer, trimer, etc.) is governed by atomic and molecular mechanics whereas the behavior of largesized clusters is governed by the macroscopic properties of the material. Therefore, as clusters form and grow, their behavior develops from the molecular to the bulk behavior.

Clusters are treated as particles so that microscopic behavior of clusters can be well represented. For simplicity, only a monatomic gas is discussed in this study. If we denote a j-mer as a cluster having $j$ atoms, then the mass of a $\mathrm{j}$-mer is $j m$. Its translational energy $\left(E_{T E}\right)$ is $j m V_{j}^{2} / 2$, where $V_{j}$ is the translational speed of the $\mathrm{j}$-mer. The average of the translational energy is then $3 k T_{j} / 2$ from gas kinetic theory, where $T_{j}$ is the temperature of the $\mathrm{j}$-mer. The internal energy $\left(E_{I E}\right)$ of a $\mathrm{j}$-mer is assumed to have an average of $3(j-1) k T_{j} / 2$ so that the total of the translational energy and internal energy of a j-mer is equal to the corresponding total of $j$ atoms. Unlike gas atoms, clusters have potential energy $\left(E_{P E}\right)$, which can be expressed as $-j h_{f g}\left(T_{j}, j\right)$. Here, $h_{f g}\left(T_{j}, j\right)$ is the latent heat of evaporation of the cluster. Clearly, $h_{f g}\left(T_{j}, j\right)$ should be small for small clusters because there are relatively few bonds for each atom; and it should approach the latent heat of evaporation of liquid $\left(h_{f g}\left(T_{j}\right)\right)$ when the cluster size increases. The latent heat of evaporation is temperature-dependent, and its relation can be derived using the first law of thermodynamics:

$$
\begin{gathered}
E_{T E}+E_{I E}+E_{P E}+Q=E_{T E 0}+E_{I E 0}+E_{P E 0} \\
\frac{3}{2} k T+\frac{3}{2}(j-1) k T-j h_{f g}(T)+j C_{p} k\left(T_{0}-T\right)=\frac{3}{2} k T_{0}+\frac{3}{2}(j-1) k T_{0}-j h_{f g}\left(T_{0}\right) \\
h_{f g}(T)=\left(C_{h}-\frac{3}{2}\right) k\left(T_{0}-T\right)+h_{f g}\left(T_{0}\right)
\end{gathered}
$$

where $Q$ is the heat required to heat the cluster from $T$ to $T_{0}$. The dependence of the latent heat on cluster size is still unclear. If we refer to the often used binding energy expression $E_{b}(j)=a_{v} j+a_{s} j^{2 / 3}\left(a_{v}\right.$ and $a_{s}$ are constants corresponding to the volume and surface terms), ${ }^{19}$ the latent heat of evaporation of clusters is assumed as follows:

$$
E_{P E}(T, j)=-j h_{f g}(T)\left(1-C_{a} j^{-1 / 3}+C_{b} j^{-1}\right)
$$

where the last term is added to increase options for parametric studies. If clusters are assumed as spheres, the radius is therefore $j^{1 / 3} r_{1}$ based on the volume. In fact, the radius of clusters is better represented by

$$
r_{j}=A j^{1 / 3}+B
$$

as predicted by molecular dynamics simulation, ${ }^{27}$ where $A$ and $B$ are constants. Molecular dynamics simulations also show that the cluster radius depends on the relative collision velocity as in the variable hard sphere molecular model: ${ }^{17}$

$$
r=r_{\text {ref }}\left(\frac{V_{r e f}}{V_{r}}\right)^{v}
$$

where $v$ is the temperature index of the viscosity, which also depends on the cluster size. ${ }^{27}$

\section{B. Nucleation}

As discussed in Section II, there is no general kinetic expression for gas nucleation. The expression from the classical nucleation theory is then used to derive a microscopic expression for the nucleation rate. A multiplicative factor may be used in the expression, and parametric studies may be performed to study effects of this factor. Here, we assume that the formation of dimers is the onset of nucleation as in general kinetic approaches. For simplicity, 
the third particle is also omitted and the metastable collision complex is assumed stabilized upon the next collision. This assumption is acceptable since we do not really know what the value of the nucleation rate is. The nucleation rate is then converted to a nucleation probability of binary collisions. Namely, a nucleation event occurs according to the probability of nucleation, which is derived as follows:

$$
P_{N}=\frac{C_{n} \sqrt{8 m \sigma / \pi}}{\rho_{l}\left(\sigma_{T} V_{r}\right)} \exp \left(-\frac{4 \pi r_{*}^{2} \sigma}{3 k T}\right)
$$

where $\sigma_{T}$ is the collision cross section.

After a nucleation event occurs, the status of the formed dimer can be evaluated as follows:

$$
\begin{gathered}
m V_{1}+m V_{1^{\prime}}=2 m V_{2} \\
\frac{1}{2} m V_{1}^{2}+\frac{1}{2} m V_{1^{\prime}}^{2}=\frac{1}{2} 2 m V_{2}^{2}+\frac{3}{2} k T_{2}+E_{P E}\left(T_{2}, 2\right)
\end{gathered}
$$

then

$$
V_{2}=\frac{1}{2}\left(V_{1}+V_{Y^{\prime}}\right), \frac{3}{2} k T_{2}=\frac{m}{4} V_{r}^{2}-E_{P E}\left(T_{2}, 2\right)
$$

\section{Condensation and Evaporation}

When a monomer collides with a cluster, there are three possible collision types: condensation, evaporation, and regular reflection. We combine the condensation and evaporation probabilities into one probability, and use the sign of the combined probability to indicate a possible nucleation or condensation event. The combined condensationevaporation probability is derived as:

$$
p_{c}=q_{s}-q_{s} \frac{p_{d}}{p} \sqrt{\frac{T}{T_{d}}}
$$

Here a positive value of $p_{c}$ indicates condensation, and a negative value refers to evaporation. If neither condensation nor evaporation occurs, the monomer-cluster collision is a scattering collision where full thermal accommodation is typically assumed. The status of particles after collisions are derived as follows:

Condensation

$$
\begin{aligned}
m V_{1}+j m V_{j} & =(j+1) m V_{j+1} \\
\frac{1}{2} m V_{1}^{2}+\frac{1}{2} j m V_{j}^{2}+\frac{3}{2}(j-1) k T_{j}+E_{P E}\left(T_{j}, j\right) & =\frac{1}{2}(j+1) m V_{j+1}^{2}+\frac{3}{2} j k T_{j+1}+E_{P E}\left(T_{j+1}, j+1\right)
\end{aligned}
$$

then

$$
\begin{gathered}
V_{j+1}=\frac{1}{j+1}\left(V_{1}+j V_{j}\right) \\
T_{j+1}=\frac{\left(\frac{3}{2}(j-1)+\left(C_{p}-\frac{3}{2}\right)\left(j-C_{a} j^{2 / 3}+C_{b}\right)\right) k T_{j}+\frac{1}{2} \frac{j}{j+1} m V_{r}^{2}+\left(\left(C_{p}-\frac{3}{2}\right) k T_{0}+h_{j g}\left(T_{0}\right)\right)\left(1+C_{a}\left(j^{2 / 3}-(j+1)^{2 / 3}\right)\right)}{\frac{3}{2} j k+\left(C_{p}-\frac{3}{2}\right) k\left(j+1-C_{a}(j+1)^{2 / 3}+C_{b}\right)}
\end{gathered}
$$

\section{Evaporation}

$$
\begin{aligned}
& V_{c}=V_{j+1} \\
& \left(\frac{1}{2} \frac{j}{j+1} m V_{r}^{2}+\left(\frac{3}{2}(j-1)+\left(C_{p}-\frac{3}{2}\right)\left(j-C_{a} j^{2 / 3}+C_{b}\right)\right) k T_{j}\right. \\
& =\left(\frac{3}{2} j k+\left(C_{p}-\frac{3}{2}\right) k\left(j+1-C_{a}(j+1)^{2 / 3}+C_{b}\right)\right) T_{j+1}-\left(\left(C_{p}-\frac{3}{2}\right) k T_{0}+h_{f g}\left(T_{0}\right)\right)\left(1+C_{a}\left(j^{2 / 3}-(j+1)^{2 / 3}\right)\right), j>1 \\
& \frac{1}{2} \frac{1}{2} m V_{r}^{2}=\frac{3}{2} k T_{2}+E_{P E}\left(T_{2}, 2\right), \quad j=1
\end{aligned}
$$

Reflection

$$
\begin{gathered}
V_{c}=\frac{1}{j+1}\left(V_{1}+j V_{j}\right) \\
\frac{1}{2} \frac{j}{j+1} m V_{r}^{\prime 2}+\left(\frac{3}{2}(j-1)+\left(C_{p}-\frac{3}{2}\right)\left(j-C_{a} j^{2 / 3}+C_{b}\right)\right) k T_{j}^{\prime}=\frac{1}{2} \frac{j}{j+1} m V_{r}^{2}+\left(\frac{3}{2}(j-1)+\left(C_{p}-\frac{3}{2}\right)\left(j-C_{a} j^{2 / 3}+C_{b}\right)\right) k T_{j}
\end{gathered}
$$


Note that the post-collision status is determined only for a condensation event. The status for an evaporation or reflection event is not determined. A general procedure for this undetermined status is to use the Borgnakke-Larsen model, ${ }^{17}$ which is explained in the next sub-section.

\section{Larsen-Borgnakke Model}

The Larsen-Borgnakke model is widely used in the DSMC method to redistribute total collision energy between translational and internal energy modes by sampling energy from two equilibrium distributions. Using the equalpartition principle, the distribution of energy $E_{a}$ from a total of $E_{a}+E_{b}$ can be expressed as follows:

$$
f(x)=f\left(\frac{E_{a}}{E_{a}+E_{b}}\right)=\frac{\Gamma\left(\xi_{a}+\xi_{b}\right)}{\Gamma\left(\xi_{a}\right) \Gamma\left(\xi_{b}\right)} x^{\xi_{a}-1}(1-x)^{\xi_{b}-1}
$$

where $\xi_{a}$ and $\xi_{b}$ are the numbers of degrees of freedom for $E_{a}$ and $E_{b}$, respectively. The instant value of energy $E_{a}$ is sampled using the acceptance-rejection method. ${ }^{17}$ Namely, a value of $E_{a}$ is chosen randomly between zero and $E_{a}+E_{b}$, then this value is used to calculate its probability ratio using Eq. (24) and is compared with a random

$$
\frac{P}{P_{\max }}(x)=\left(\frac{\xi_{a}+\xi_{b}-2}{\xi_{a}-1} x\right)^{\xi_{a}-1}\left(\frac{\xi_{a}+\xi_{b}-2}{\xi_{b}-1}(1-x)\right)^{\xi_{b}-1}
$$

fraction $R_{f}$ that is generated from a uniform distribution between 0 and 1 . This value of $E_{a}$ is accepted if the probability ratio is greater than $R_{f}$, but a new value is chosen and the process is repeated if the ratio is less than $R_{f}$. However, when $\xi_{a}$ and $\xi_{b}$ are not of the same order of magnitude, many attempts have to be made to find a value of $E_{a}$. A remedy is to avoid finding $x$ itself by converting $x$ to a new variable $y$ whose value is close to 0.5 by using a transformation $y=x^{1 / a}$. The value of $a$ can be chosen as $\log _{2}^{\left(\xi_{a}+\xi_{b}\right) / \xi_{a}}$. Then the distribution for $y$ is

$$
f(y)=\frac{a \Gamma\left(\xi_{a}+\xi_{b}\right)}{\Gamma\left(\xi_{a}\right) \Gamma\left(\xi_{b}\right)}\left(y^{a}\right)^{\xi_{a}-1 / a}\left(1-y^{a}\right)^{\xi_{b}-1}
$$

and the probability ratio is

$$
\frac{P}{P_{\max }}(y)=\left(\frac{\xi_{a}+\xi_{b}-1-1 / a}{\xi_{a}-1 / a} y^{a}\right)^{\xi_{a}-1 / a}\left(\frac{\xi_{a}+\xi_{b}-1-1 / a}{\xi_{b}-1}\left(1-y^{a}\right)\right)^{\xi_{b}-1}
$$

To compare the numerical performance of the standard and modified Larsen-Borgnakke models, the average number of attempts required during the acceptance-rejection procedure is plotted in Fig. 1 (a) where the percentage of one

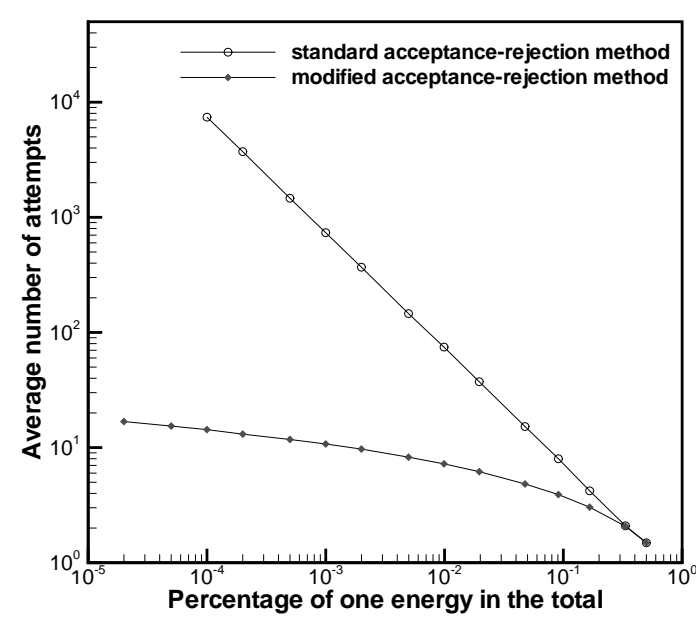

(a)

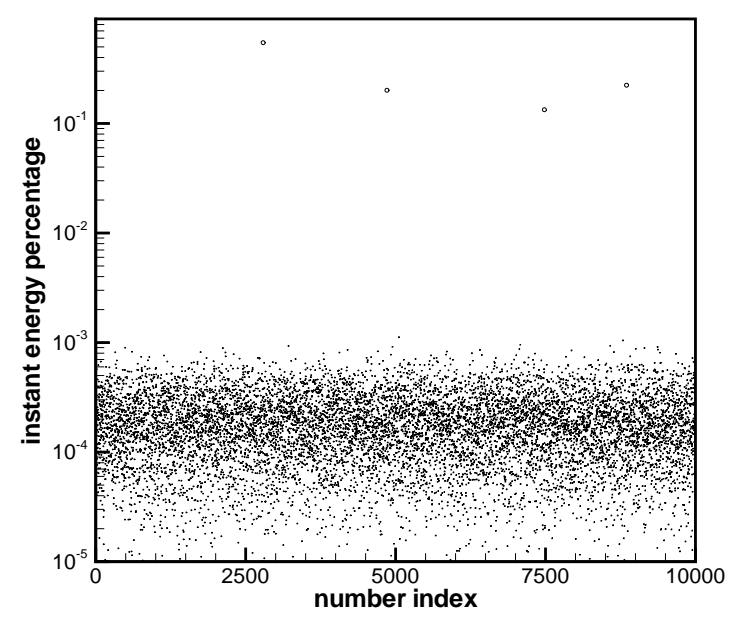

(b)

Figure 1. Numerical performance of the Larsen-Borgnakke model. (a) comparison of number of attempts during the acceptance-rejection procedure, (b) instant distribution of the energy percentage using the standard Larsen-Borgnakke method. 
energy in the total is $E_{a} /\left(E_{a}+E_{b}\right)$. Clearly, the modified Larsen-Borgnakke method improves greatly the numerical efficiency when $\xi_{a}$ is much smaller than $\xi_{b}$, which is usually the case for clusters having many atoms. Numerical tests also show that the standard Larsen-Borgnakke method can accept unusual values with a relatively large probability. For example, Fig 1(b) shows the instant energy percentage $\left(E_{a} /\left(E_{a}+E_{b}\right)\right)$ distribution whose theoretical average value is $2 \mathrm{e}^{-4}$. We find that there are 4 values larger than 0.1 among 10,000 values, which is statistically too much. These undesired values, however, are avoided when the modified Larsen-Borgnakke method is employed (the plot is otherwise similar to Fig. 1(b), and is therefore not shown).

\section{Parametric Studies}

The developed kinetic model is implemented in the DSMC research code "MONACO". ${ }^{28}$ The clusters are modeled as particles in a similar way to monomers. Clusters have translational velocity; and their internal and potential energy is represented by the cluster temperature. The sub-relaxation technique ${ }^{29}$ is employed to evaluate the macroscopic properties (gas temperature, pressure, et al.) that are explicitly involved in the developed microscopic model. We use argon gas to illustrate the nucleation and condensation processes.

As discussed in Section III, there are many uncertainties about the nucleation and condensation processes. For simplicity, the clusters are modeled as hard spheres, which means that the cluster radius is calculated as $j^{1 / 3} r_{1}$. The surface tension of clusters is approximated as $0.0344\left(1-T / T_{c}\right) \mathrm{N} / \mathrm{m}$ following Hale, ${ }^{30}$ where the critical temperature $\left(T_{c}\right)$ is about $150.85 \mathrm{~K}$. The saturated vapor pressure $P_{s}$ is used as $P_{d}$ for the evaporation calculation. The saturated vapor pressure is written using a logarithmic-exponential curve-fit expression as follows:

$$
\ln P_{s}=-396.465+225.279 \ln T-41.7722 \ln ^{2} T+2.6374 \ln ^{3} T
$$

when the temperature is in the range between $20 \mathrm{~K}$ and $150.85 \mathrm{~K}$.

Two numerical examples are employed to study effects of several parameters on gas nucleation and condensation. One is a box flow. The argon gas represented by 90,000 particles is simulated in a square box where the box surface is assumed specular. The initial number density and temperature are $1 \times 10^{26} / \mathrm{m}^{3}$ and $55 \mathrm{~K}$, respectively. Other parameters are set as following unless specified otherwise: $C_{n}=10^{8}, q_{s}=0.1, C_{a}=2.0$, and $C_{b}=1.175$. The second example involves supersonic flow in an expansion nozzle.

\section{A. Nucleation Rate}

There is not much information about the nucleation rate at low temperature. It is therefore very important to study effects of this rate on flow properties. Figure 2 shows some plots using three different values for the rate constant. In this study, evaporation of a dimer to two monomers is disabled because fluctuation of flow properties cannot nucleate a dimer after a dimer is accidentally evaporated. These plots show that the number of clusters increases linearly at the early time and then quickly reaches a constant as the degree of supersaturation decreases because the monomers are converted into clusters and gas temperature is increased. The average cluster size is almost independent of the nucleation rate at very early time, but then increases in a behavior dominated by the condensation process (smaller nucleation rate corresponds to larger clusters). Both gas and cluster temperatures increase as more and more gas is converted into clusters, and the gas temperature lags behind the cluster temperature. It takes more than a tenth of a microsecond to reach the steady state. In general, a larger nucleation rate means that there will be more clusters but having a smaller average size, and the gas will be heated quicker but there is less effect on the final flow temperature which is basically determined by the vapor pressure.

\section{B. Sticking Coefficient}

The sticking coefficient is usually pre-assumed for condensation simulations. Recent studies of Zhong et al. ${ }^{27}$ showed that the sticking coefficient depended on the cluster size. For the time being, we only consider a fixed sticking coefficient for all clusters. Figure 3 shows comparisons of several flow properties using three different values for the sticking coefficient. Clearly, the sticking coefficient has no effect on the number of clusters at early time, but a larger sticking coefficient means a large cluster size and later limits the number of clusters at steady state. Because the sticking coefficient determines the condensation rate, it also affects the temperature of both gas and clusters. A larger sticking coefficient will heat the gas more quickly. If the sticking coefficient is very small, then gas-cluster collisions will have a larger possibility for a reflection collision; so the gas temperature can closely follow the cluster temperature. 


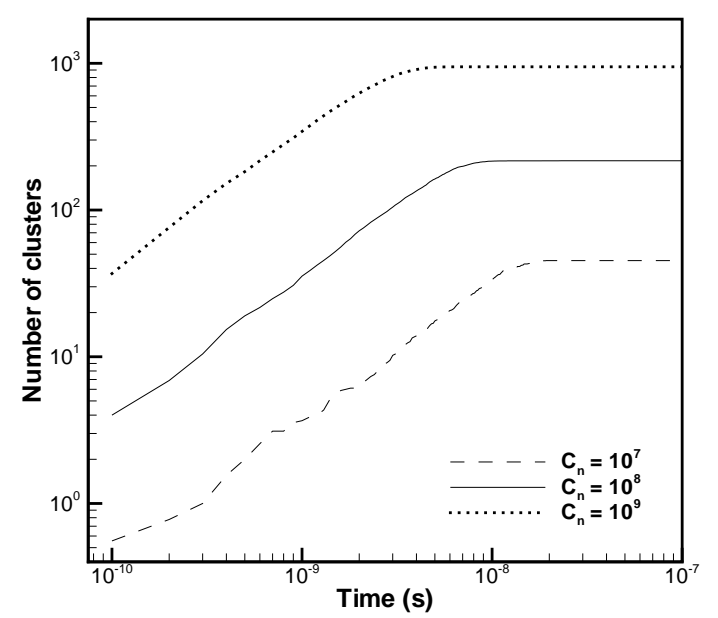

(a)

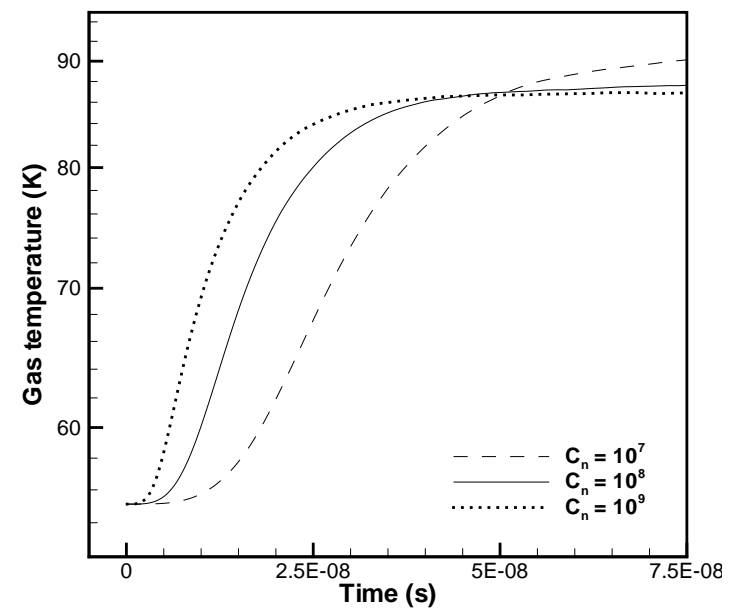

(c)

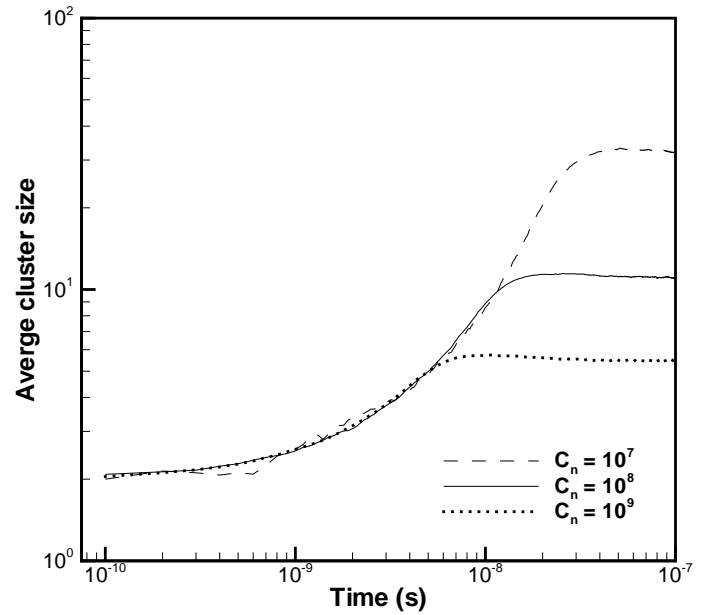

(b)

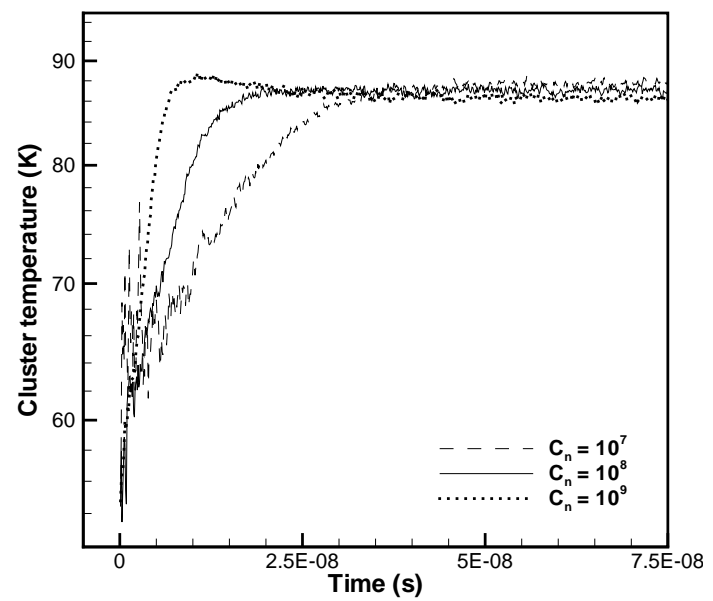

(d)

Figure 2. Effects of nucleation rate on flow properties. (a) number of clusters, (b) average cluster size, (c) gas temperature, (d) cluster temperature.

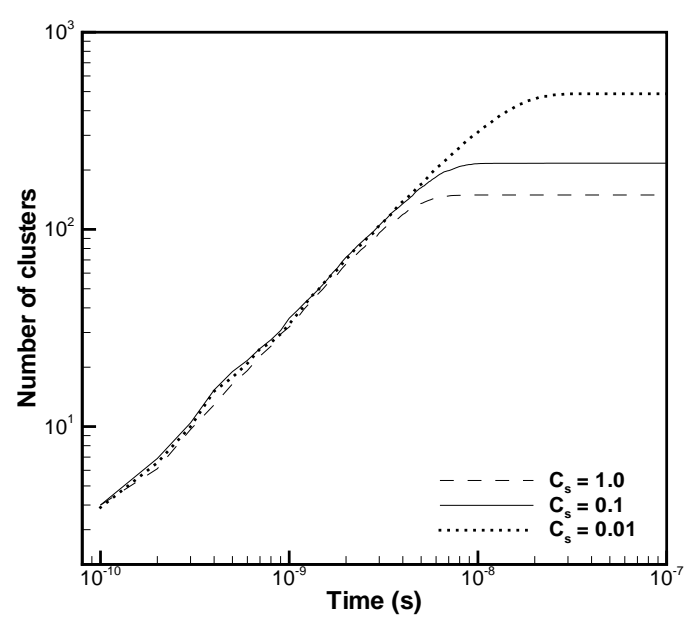

(a)

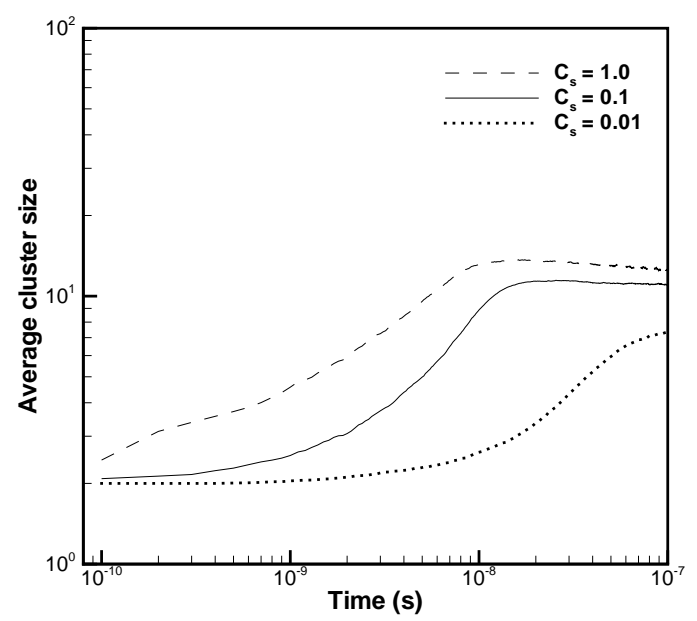

(b) 


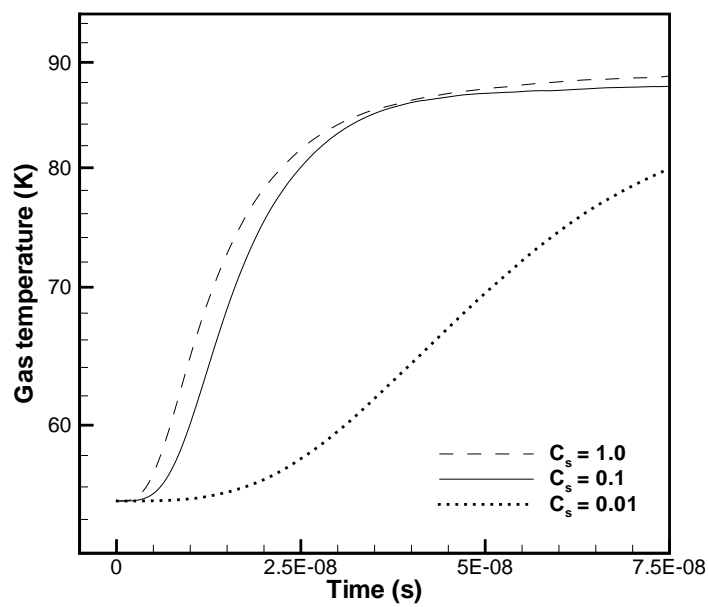

(c)

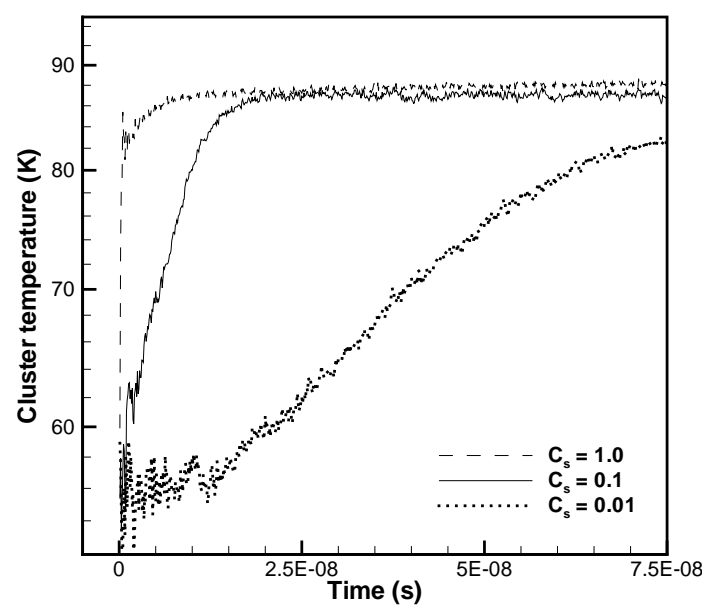

(d)

Figure 3. Effects of sticking coefficient on flow properties. (a) number of clusters, (b) average cluster size, (c) gas temperature, (d) cluster temperature.

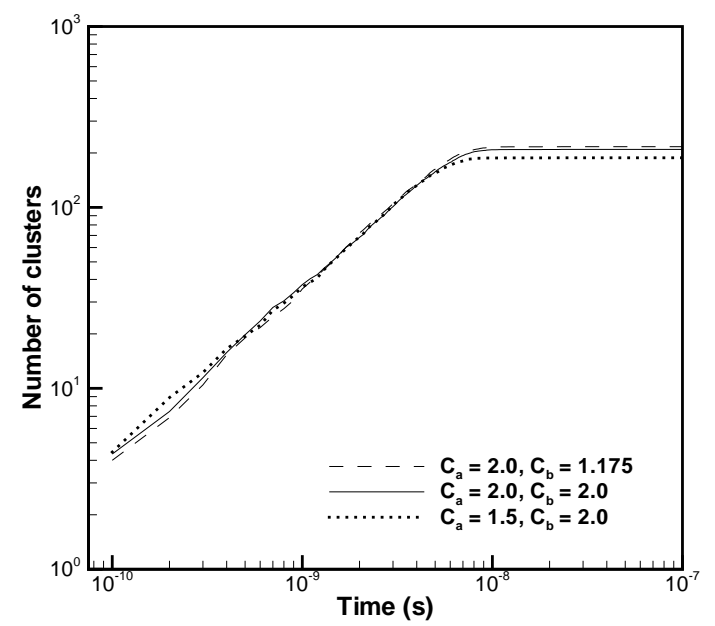

(a)

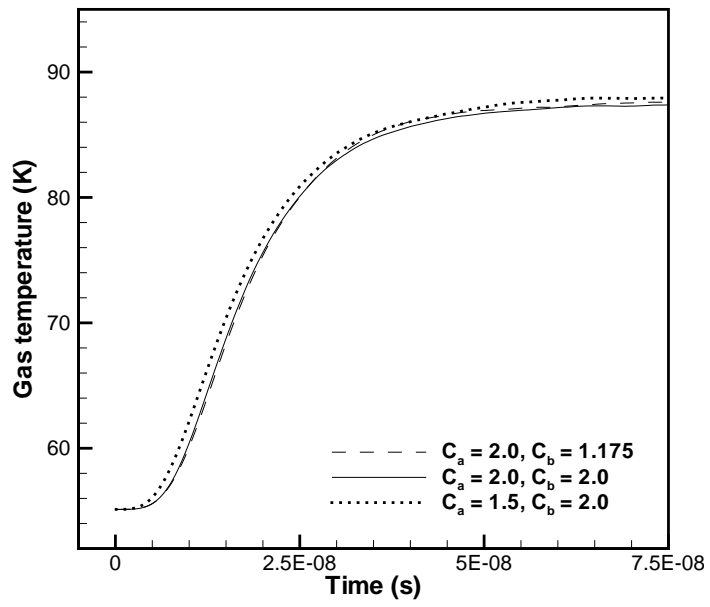

(c)

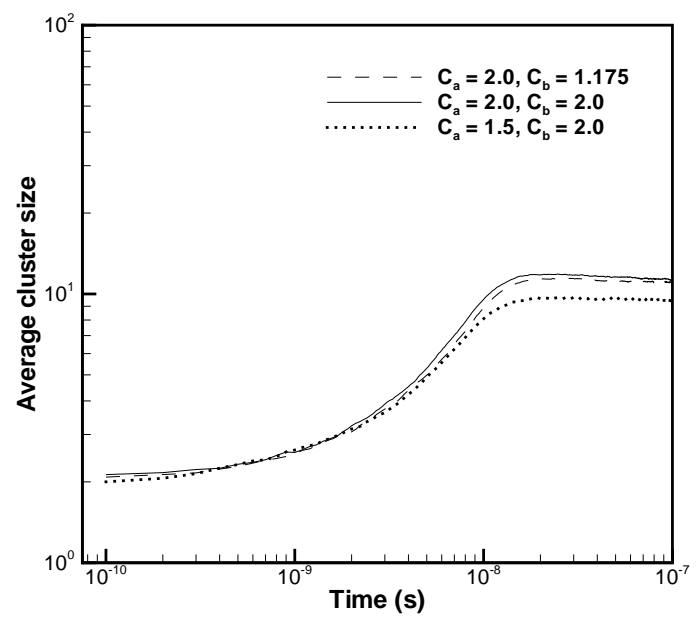

(b)

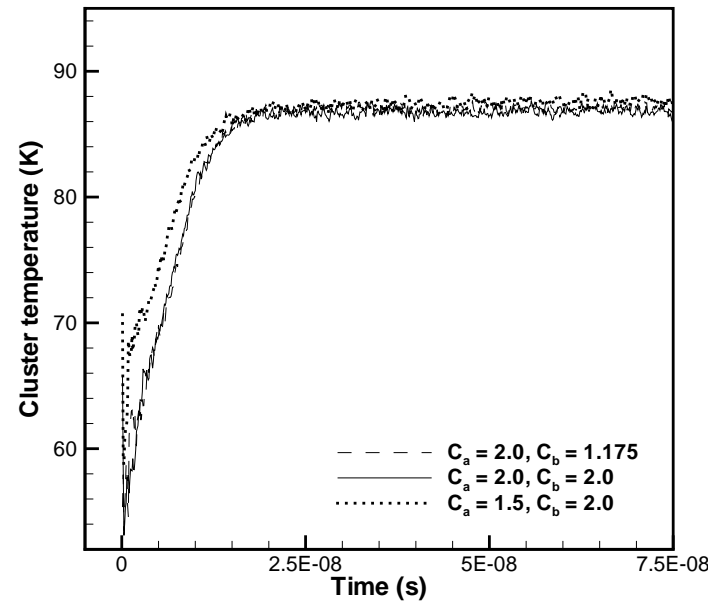

(d)

Figure 4. Effects of potential energy on flow properties. (a) number of clusters, (b) average cluster size, (c) gas temperature, (d) cluster temperature. 


\section{Potential Energy}

The value of potential energy is also unclear for small clusters. We therefore test effects of potential energy on flow properties. Figure 4 shows some results using several different values for the potential energy. Maybe because of the small sticking coefficient (0.1), there are no large effects of the potential energy tested on the flow properties. Further studies are required to draw solid conclusions for effects of the potential energy.

\section{Supersonic Flow in Expansion Nozzle}

It is not unusual that gas nucleation and condensation occurs in supersonic flow in expansion nozzles. ${ }^{421-22} \mathrm{We}$ use a simple example to illustrate the gas nucleation and condensation phenomena in supersonic expansion flows. The expansion nozzle is two dimensional, and the surface is assumed specular. The inflow argon gas has a uniform velocity of $400 \mathrm{~m} / \mathrm{s}$, a temperature of $60 \mathrm{~K}$, and a number density of $10^{24} / \mathrm{m}^{3}$. Values of several parameters used in the developed kinetic model are set as follows: $C_{n}=10^{28}, q_{s}=1.0, C_{a}=2.0$, and $C_{b}=1.175$.

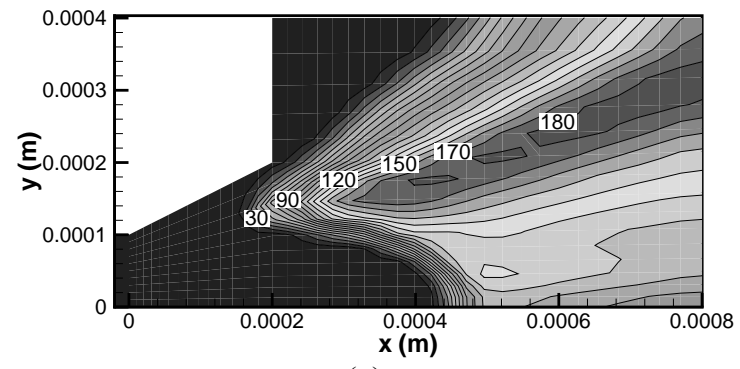

(a)

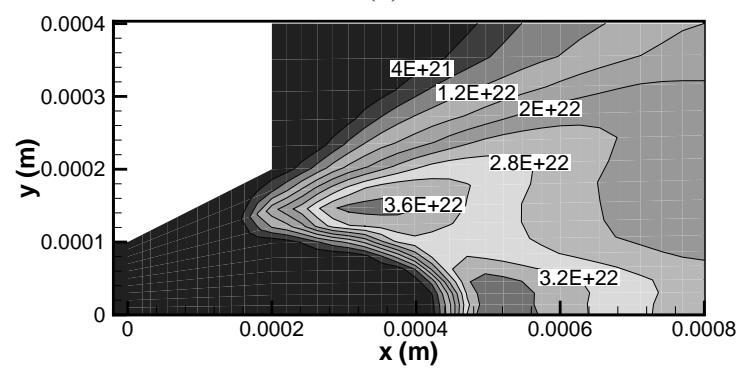

(c)

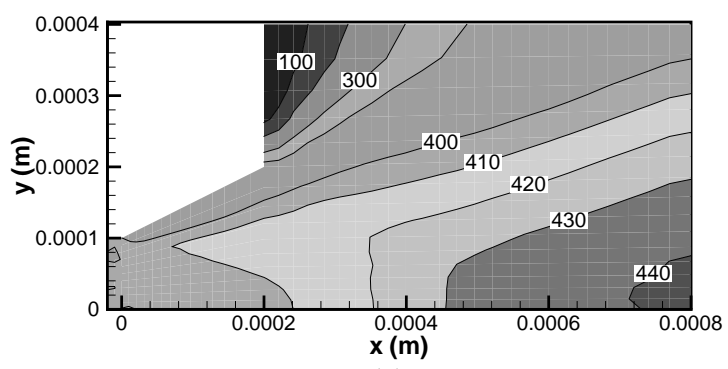

(e)

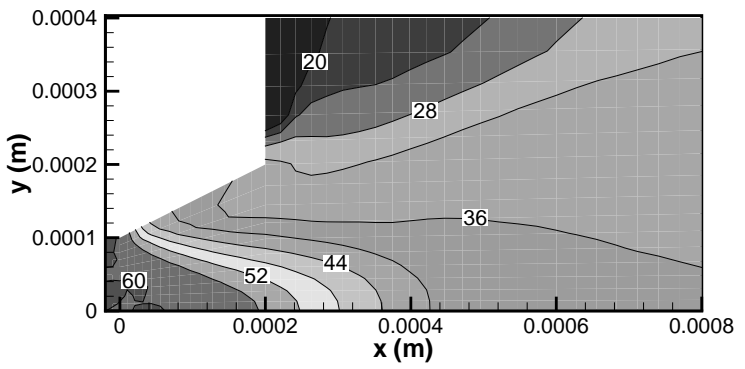

(g)

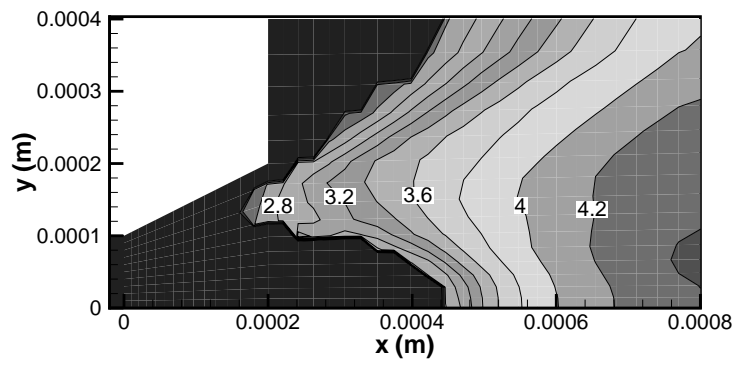

(b)

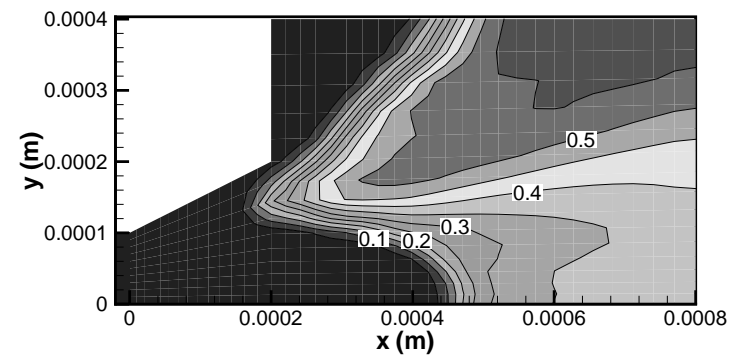

(d)

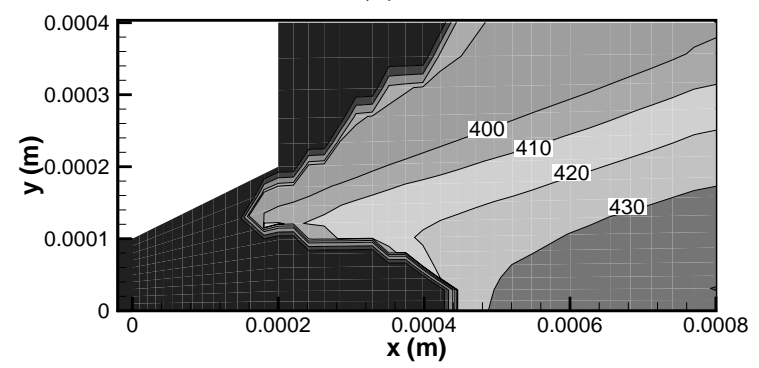

(f)

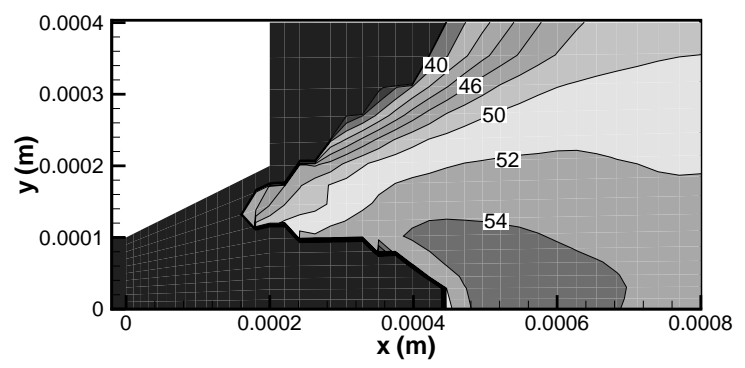

(h)

Figure 5. Supersonic flow in an expansion nozzle. (a) number of clusters, (b) average cluster size, (c) number density of clusters $\left(1 / \mathrm{m}^{3}\right)$, (d) mass fraction of clusters, (e) gas velocity $(\mathrm{m} / \mathrm{s})$, (f) cluster velocity $(\mathrm{m} / \mathrm{s})$, (g) gas temperature (K), (h) cluster temperature $(K)$. 
The flow patterns of this problem are illustrated in Fig. 5. Figure 5(a) shows the number of clusters formed in the simulated domain (the average particle number in each cell is about 500). Clearly, the clusters are formed in a relatively small region (Fig. 5(c)), and the cluster size increases as the clusters move downstream in the flow (Fig. 5(b)). So the mass fraction of clusters also increases downstream the flow (Fig. 5(d)). It is found that the velocity of clusters (Fig. 5(f)) is very close to the gas velocity (Fig. 5(e)), whereas the cluster temperature (Fig. 5(h)) is larger than the gas temperature (Fig. 5(g)) because there are few reflection collisions between gas molecules and clusters. If we decrease the sticking coefficient, then few gas molecules will condense onto each cluster (Fig. 6(b)). However, there are more clusters formed in the flow (Fig. 6(a)). The temperature of both gas and cluster decreases, and the difference between them also decreases. If we decrease the nucleation rate $\left(C_{n}=10^{22}\right)$, then clusters are formed further downstream of the nozzle (Fig. 7(a)) where the degree of supersaturation is larger. The average size of clusters increases, and the temperature of both gas and clusters decreases as expected.

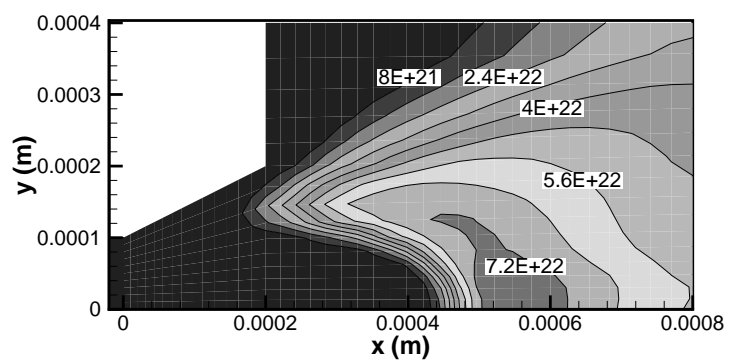

(a)

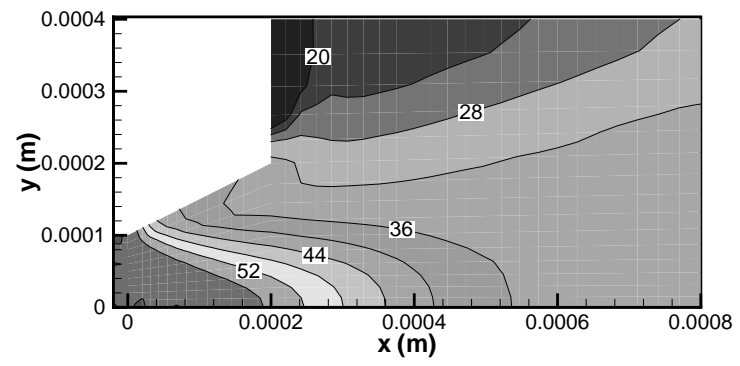

(c)

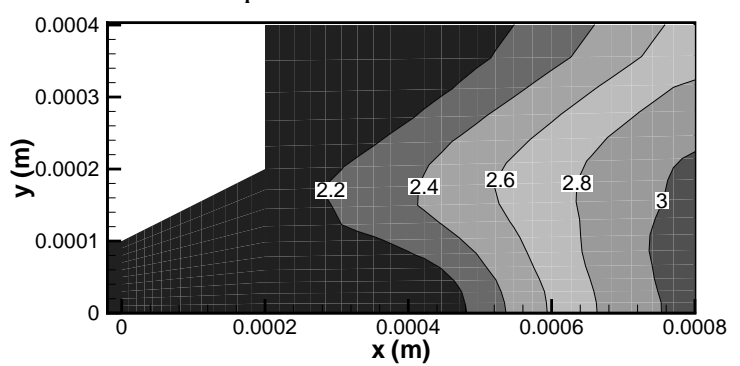

(b)

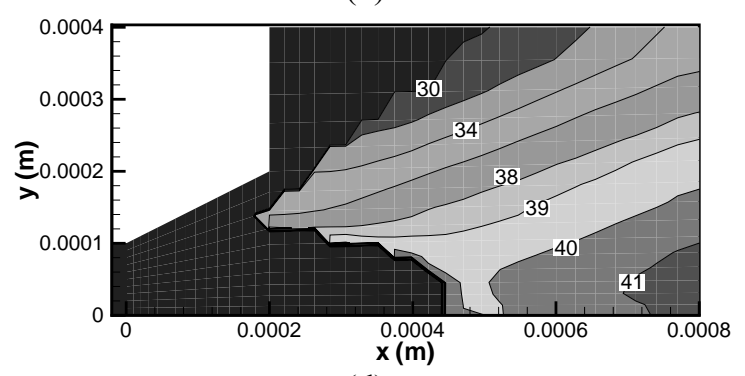

(d)

Figure 6. Supersonic flow in an expansion nozzle with a smaller sticking coefficient (0.1). (a) number of clusters, (b) average cluster size, (c) gas temperature (K), (d) cluster temperature $(K)$.

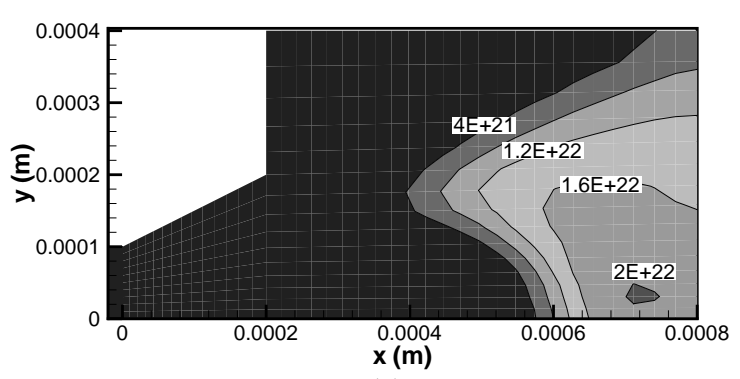

(a)

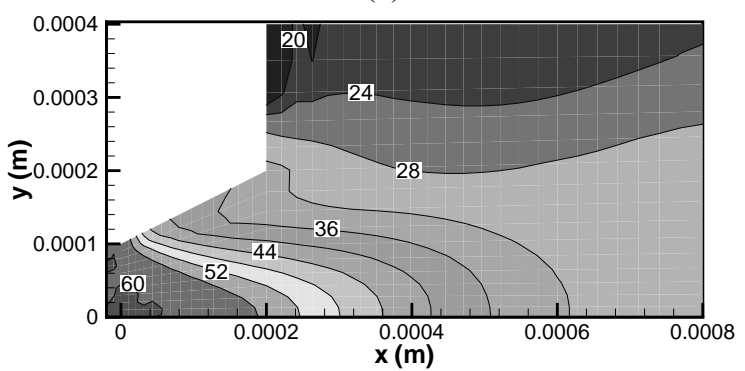

(c)

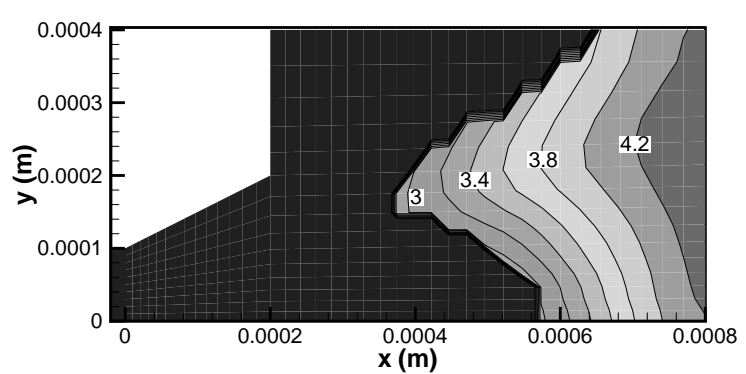

(b)

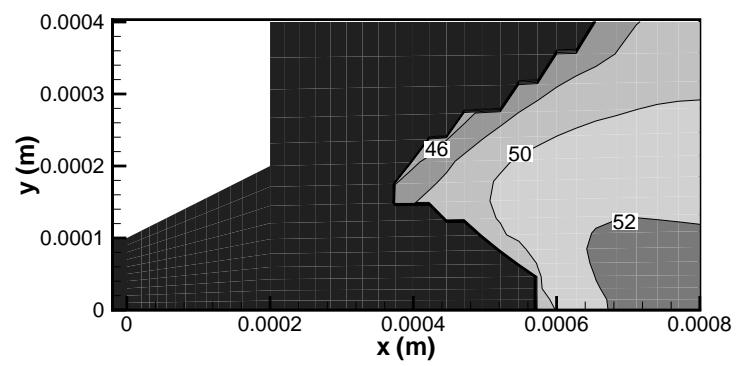

(d)

Figure 7. Supersonic flow in an expansion nozzle with a smaller nucleation rate. (a) number of clusters, (b) average cluster size, (c) gas temperature $(K),(d)$ cluster temperature $(K)$. 


\section{Discussion and Conclusion}

Gas nucleation and condensation is very common in both nature and industry. There have been many studies on this topic. The classical nucleation theory is able to explain some physics of the nucleation processes, and molecular dynamics simulation is supposed to expose the detailed mechanism of gas nucleation and condensation. However, due to the physical inaccuracy of the classical nucleation theory for small clusters and the extremely expensive numerical cost for molecular dynamics simulations, there is no general tool for studying gas flows having clusters.

The direct simulation Monte Carlo method is a less numerically expensive but still kinetically accurate method. We develop a general nucleation and condensation model and implemented it in the DSMC method, and thus detailed flow simulation becomes possible for flows involving clusters. In this model, clusters are modeled as particles, but both internal energy and potential energy are considered. Gas nucleation and condensation are modeled via particle collisions. The probability for possible nucleation or condensation, however, cannot be determined from the model itself. Since there are many uncertainties in the physics of nucleation and condensation, the DSMC method is very useful to investigate effects of parameters on general flow properties.

Our parametric studies showed that the proposed model was able to test effects of different parameters. The nucleation rate affected not only the number of clusters and cluster size but also the properties of the remaining gas. Investigation also showed that the sticking coefficient was very important for both cluster and gas properties. The application of the proposed model to a model example, supersonic flow in an expansion nozzle, demonstrated the capability of the proposed model: it is able to simulate complicated flows involving clusters and can predict detailed flow properties of both gas and clusters.

\section{Acknowledgments}

This work was supported by the Air Force Office of Scientific Research under grant F49620-03-1-0123. The views and conclusions contained herein are those of the authors and should not be interpreted as necessary representing the official policies or endorsements, either expressed or implied, of the AFOSR or the U.S. Government.

\section{References}

${ }^{1}$ Zhang, R, et al., “Atmospheric New Particle Formation Enhanced by Organic Acids,” Science, Vol. 304(5676), 2004, pp. 1487-1490.

${ }^{2}$ Urban, D.L., and Faeth, G.M., "Soot Research in Combustion Science: Introduction and Review of Current Work," AIAA 2001-0322, 2001.

${ }^{3}$ Wu, B.J., "Possible Water Vapor Condensation in Rocket Exhaust Plumes," AIAA Journal, Vol. 13(6), 1975, pp. 797-802.

${ }^{4}$ Hill, P.G., "Condensation of Water Vapour During Supersonic Expansion in Nozzles," Journal of Fluid Mechanics, Vol. 25(3), 1966, pp. 593-620.

${ }^{5}$ McIlroy, D.N., et al., "Nanoparticle Formation in Microchannel Glass by Plasma Enchanced Chemical Vapor Deposition," Journal of Applied Physics, Vol. 93(9), 2003, pp. 5643-5649.

${ }^{6}$ Selwyn, G.S., Singh, J., and Bennette, R.S., "Insitu Laser Diagnostic Studies of Plasma-Generated Particulate Contamination," Journal of Vacuum Science \& Technology A, Vol. 7(4), 1989, pp. 2758-2765.

${ }^{7}$ Liu. S., Xie, E., Sun, J., Ning, C., and Jiang, Y., "Astudy on Nano-Nucleation and Interface of Diamond Film Prepared by Hot Filament Assisted with Radio Frequency Plasma,” Materials Letters, Vol. 57(11), 2003, pp. 1662-1669.

${ }^{8}$ Abraham, F. F., Homogeneous Nucleation Theory, Academic Press, 1974.

${ }^{9}$ Oxtoby, D. W., "Homogeneous Nucleation: Theory and Experiment," Journal of Physics: Condensed Matter, Vol. 4, 1992, pp. 7627-7650.

${ }^{10}$ Lothe, J., and Pound, G.M., "Reconsiderations of Nucleation Theory," Journal of Chemical Physics, Vol. 36(8), 1962, pp. 2080-2085.

${ }^{11}$ Hettema, H., and McFeaters, J. S., "The Direct Monte Carlo Method Applied to the Homogeneous Nucleation Problem," Journal of Chemical Physics, Vol. 105 (7), 1996, pp. 2816-2827.

${ }^{12}$ Toxvaerd, S., "Molecular Dynamics Simulations of Nucleation," Molecular Simulation, Vol. 30(2-3), 2004, pp. $179-182$.

${ }^{13}$ Zurek, W.H., and Schieve, W.C., "Molecular Dynamics Study of Clustering. I," Journal of Chemical Physics, Vol. 68(3), 1978, pp. 840-846.

${ }^{14}$ Weiss, S., "Dimer and Trimer Formation in Dense Gaseous Argon: A MD Study," Journal of Physical Chemistry A, Vol. 101(18), 1997, pp. 3367-3370.

${ }^{15}$ Soto, R., and Cordero, P., "Cluster Birth-Death Processes in a Vapor at Equilibrium," Journal of Chemical Physics, Vol. 110(15), 1999, pp. 7316-7325.

${ }^{16}$ Toxvaerd, S., "Molecular Dynamics Simulations of Nucleation,” Molecular Simulation, Vol. 30(2-3), 2004, pp. $179-182$.

${ }^{17}$ Bird, G. A., Molecular Gas Dynamics and the Direct Simulation of Gas Flows, Oxford University Press, 1994.

${ }^{18}$ Briehl, B., and Urbassek, H. M., "Monte Carlo Simulation of Growth and Decay Processes in a Cluster Aggregation Source," Journal of Vacuum Science and Technology A, Vol. 17(1), 1999, pp. 256-265. 
${ }^{19}$ Mizuseki, H, Jin, Y., Kawazoe, Y., and Wille, L.T., "Growth Processes of Magnetic Clusters Studied by Direct Simulation Monte Carlo Method," Journal of Applied Physics, Vol. 87(9), 2000, pp. 6561-6563.

${ }^{20}$ Insepov, Z., and Yamada, I., "Direct Simulation Monte Carlo Method for Gas Cluster Ion Beam Technology", Nuclear Instruments and Methods in Physics Research B, Vol. 202, 2003, pp. 283-288.

${ }^{21}$ Zhong J., Gimelshein S. F., Zeifman, M. I., and Levin, D. A., "Modeling of Homogeneous Condensation in Supersonic Plumes with the DSMC Method," AIAA 2004-0166, 2004.

${ }^{22}$ Sun, Q., Boyd, I.D., and Tatum, K.E., "Particle Simulation of Gas Expansion and Condensation in Supersonic Jets," AIAA 2004-2587, 2004.

${ }^{23}$ Perrell, E. R., Erickson, W. D., and Candler, G. V., "Numerical Simulation of Nonequilibrium Condensation in a Hypersonic Wind Tunnel," Journal of Thermophysics and Heat Transfer, Vol. 10(2), 1996, pp. 277-283.

${ }^{24}$ Itkin, A.L., "Kinetic Model of Coupled Nonequilibrium Condensation and Radiative Excitation of Water Molecules," Journal of Chemical Physics, Vol. 108(9), 1998, pp. 3660-3677.

${ }^{25}$ Hillenkamp, M., Keinan, S., and Even, U., "Condensation Limited Cooling in Supersonic Expansions," Journal of Chemical Physics, Vol. 118(19), 2003, pp. 8699-8705.

${ }^{26}$ Zeifman, M.I., Zhong, J., and Levin, D.A., "A Hybrid MD-DSMC Approach to Direct Simulation of Condensation in Supersonic Jets," AIAA 2004-2586, 2005.

${ }^{27}$ Zhong, J, Zeifman, M.I., and Levin, D.A., “A Kinetic Model of Condensation in a Free Argon Expanding Jet," AIAA 2005767, 2005.

${ }^{28}$ Dietrich, S., and Boyd, I.D., "Scalar and Parallel Optimized Implementation of the Direct Simulation Monte Carlo Method," Journal of Computational Physics, Vol. 126, 1996, pp. 328-342.

${ }^{29}$ Sun, Q., and Boyd, I.D., "Evaluation of Macroscopic Properties in the Direct Simulation Monte Carlo Method," Journal of Thermophysics and Heat Transfer (to be published).

${ }^{30}$ Hale, B.N., "Monte Carlo Calculations of Effective Surface Tension for Small Clusters," Austrian Journal of Physics, Vol. 49, 1996, pp. 425-434. 